\title{
Dynamic control of DEP actuation and droplet dispensing
}

\author{
K-L Wang ${ }^{1}$, T B Jones ${ }^{1}$ and A Raisanen ${ }^{2}$ \\ ${ }^{1}$ University of Rochester, Rochester, NY 14627 USA \\ ${ }^{2}$ Rochester Institute of Technology, Rochester, NY 14623 USA \\ E-mail: jones@ece.rochester.edu
}

Received 13 October 2006, in final form 8 November 2006

Published 5 December 2006

Online at stacks.iop.org/JMM/17/76

\begin{abstract}
Rapid dielectrophoretic (DEP) liquid actuation and dispensing of uniform aqueous droplets on a substrate are implemented for the first time in a silicon-based architecture. Using $\langle 100\rangle$ Si wafers takes advantage of the high thermal conductivity and extensive semiconductor microfabrication capabilities of silicon. The structures employ a coplanar, three-electrode design to guarantee reproducible dispensing of multiple sessile droplets as small as $\sim 75 \mathrm{pl}$. A reduced-order dynamic model provides a predictive relation for the transient liquid motion, which is implemented in an open-loop dynamic control scheme to achieve repeatable production of anywhere from 1 to 23 droplets per structure by controlling voltage on-time.
\end{abstract}

(Some figures in this article are in colour only in the electronic version)

\section{Introduction}

The lab-on-a-chip (LOC) integrates microfluidic control and sensing with automated chemical or biochemical analyses and diagnostics. Such systems may someday alleviate bottlenecks associated with performing large numbers of repetitive protocols. In addition to greater speed and lower cost, the automated LOC also offers the advantages of lowered risk of human exposure to dangerous pathogens and reduced consumption of expensive chemicals and bioagents.

Many microfluidic schemes utilize electrical forces to actuate flow: e.g., electrocapillarity [1], electro-osmosis [2], electroconvection [3] and electrowetting [4]. Electrowettingon-dielectric (EWOD) [5] and liquid dielectrophoresis (DEP) [6], exemplifying electromechanical microfluidic systems [7], show to best advantage in architectures where the basic unit for transport and processing is the droplet. Schemes using volumes ranging from $\sim 1 \mu$ l down to $\sim 10 \mathrm{pl}$ have been demonstrated. In this paper, we report a DEPbased droplet dispenser fabricated on a $\langle 100\rangle$ crystalline Si wafer. A significant advantage of Si substrates is that, unlike glass, a host of fabrication methodologies from the semiconductor industry can be exploited to create complex structures integrating essential electronics and sensor systems with the microfluidics on a single chip. Furthermore, the thermal conductivity of Si is very high, thereby facilitating improved temperature control.

\section{DEP droplet dispensing}

DEP microfluidics features high-speed mass transport (10 $10^{2} \mathrm{~cm} \mathrm{~s}^{-1}$ ) and the capability to dispense large numbers of aqueous droplets ranging from $\sim 10^{2} \mathrm{nl}$ down to $\sim 10 \mathrm{pl}$ [8]. The dispenser shown in figure $1(a)$ is an open structure consisting of parallel, coplanar electrodes patterned on an insulating substrate and coated with a dielectric layer of thickness $d$. To prevent electrolysis and to promote smooth motion, the electrodes are coated with dielectric and hydrophobic layers.

In operation, a large liquid drop is manually deposited with a micropipette on the right side of the two-electrode structure as shown by the dashed circle in figure $1(a)$. Applying voltage to the electrodes forms a finger-shaped liquid rivulet with semicircular cross-section, which protrudes from the manually dispensed parent drop and moves rapidly along the electrodes. The liquid finger has a semicircular profile of diameter $D=2 w+g$, where $w$ is electrode width and $g$ is gap spacing. The rivulet stops when it gets to the end of the electrodes and remains in a stable, electrohydrostatic equilibrium until the voltage is removed ${ }^{3}$. When voltage is turned off, capillary instability takes over to form sessile droplets within $2 \mathrm{~ms}$. Reliable droplet placement

3 DEP actuation, analogous to capillarity in that it leads to a new hydrostatic equilibrium, strictly speaking, is not a true pumping mechanism. 

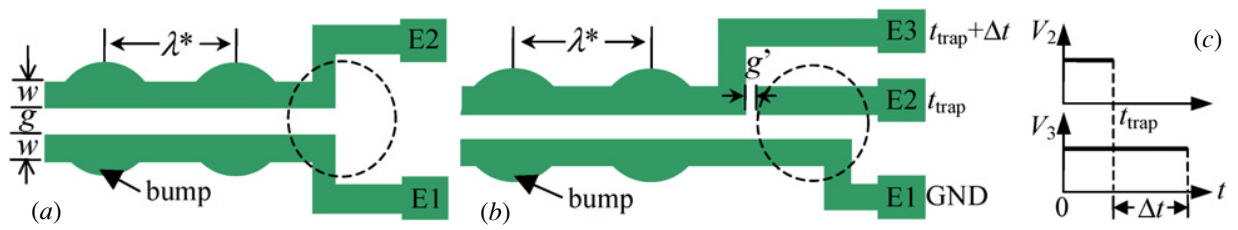

Figure 1. (a) DEP droplet dispenser showing the original two-electrode design. The bumps are spaced at the most unstable wavelength $\lambda^{*}$. (b) Three-electrode coplanar structure with a T-junction gap $g^{\prime}$ to trap liquid along E3. Dashed circles (not to scale) indicate locations where parent drop is initially deposited with the micropipette. (c) Timing for voltage application on electrodes E2 and E3.

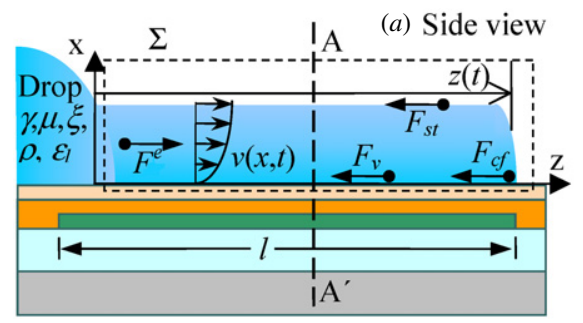

\section{(b) $\mathrm{A}-\mathrm{A}^{\prime}$ cross-section}

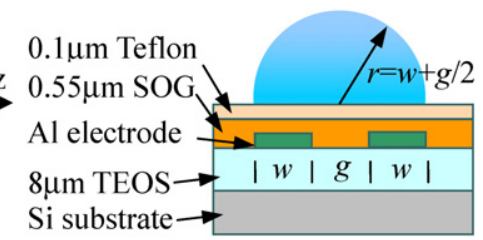

Figure 2. (a) Side view of liquid finger showing the control volume $\Sigma$ (dashed line). DEP force $F^{e}$ overcomes capillarity $F_{\text {st }}$, to initiate

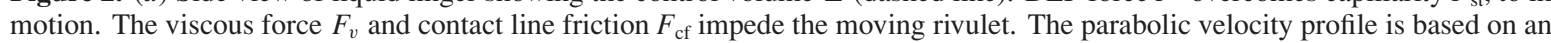
assumption of laminar flow with a non-slip condition at the liquid/substrate interface. (b) Cross section of coplanar structure with semicircular finger profile. Al electrodes are fabricated on a TEOS-passivated Si wafer and spin-coated with SOG and Teflon AF. Drawing is not to scale.

and size uniformity are promoted by the periodic semicircular bumps centered on the electrode midline. The bump size is determined assuming the hemispherical profile of uniform droplets, and the optimal bump spacing corresponds closely to the most unstable wavelength for the capillary jet instability, i.e., $\lambda^{*}=4.508 D[9,10]$.

A critical shortcoming observed with the two-electrode structure is that, depending on net width $D$, surface tension pulls $30 \%$ or more of the liquid back into the parent drop after voltage removal [8]. This back-flow influences the hydrodynamic instability and leads to irregularly sized and spaced droplets. To solve this problem, the original twoelectrode design was modified by dividing one of the electrode strips into two sections, as shown in figure $1(b)$. In this new three-electrode design, voltage is applied initially on both E2 and E3. The liquid finger forms and starts to move, jumps the transverse gap $g^{\prime}$, flows to the end of the structure and then stops. Next, voltage is removed from E2, trapping liquid along the entire length between E1 and E3. When voltage is now removed from E3, there is no backflow and regularly spaced, uniform droplets form. For example, structures of dimensions $w / g / g^{\prime}=13 / 9 / 5 \mu \mathrm{m}$ have been used successfully to produce as many as 32 uniform droplets of volume $\sim 75 \mathrm{pl}$ each. There is every reason to expect that longer structures can be used to form 50 or more droplets per electrode pair. Scaling up this scheme to large planar arrays of sessile droplets is readily envisioned.

\section{Hydrodynamic model}

When sufficient voltage is applied to the dielectric-coated coplanar electrodes, the surface tension of the sessile parent drop is overcome and a finger-shaped rivulet protrudes and moves along the structure. Figure 2(a) shows a side view of the moving finger on the co-planar, dielectric-coated electrodes, which are of length $l$, width $w$ and spacing $g$. The condition $w, g \ll l$ assures that the imposed electric field is uniform along the electrodes, while the condition on the dielectric layer thickness $d \ll w, g$ promotes the uniformity of the semicircular finger profile depicted in figure $2(b)$.

The hydrodynamic model is derived from imposing conservation of momentum on the control volume $\Sigma$ shown by the dashed rectangle in figure $2(a)$. The driving force of electrical origin can be determined from a capacitive model using classical methods of lumped parameter electromechanics. Refer to the appendix for a summary of the derivation of $F^{e}$ :

$$
F^{e}=\frac{1}{2} \frac{\mathrm{d} C}{\mathrm{~d} z} \cdot V^{2}
$$

Here, $C(z)$ is the total capacitor measured between the coplanar electrodes and $z$ is the time-dependent finger length. If it is assumed that the finger cross section is uniform along its length and does not change in time, then the DEP force will be constant throughout the transient motion, until the finger reached the end of the structure. It may be shown that the electrode width $w$ and spacing $g$ have only a minor influence on the capacitance $C$.

Impeding the finger motion are the capillary force, $F_{\text {st }}$, due to surface tension, the viscous force, $F_{v}$, due to shear stress at the liquid/dielectric interface, and another friction force, $F_{\mathrm{cf}}$, related to the forward motion of the advancing contact line $[11,12]$. The capillarity force is

$$
F_{\text {st }}=-\pi(w+g / 2) \gamma
$$

where $\gamma$ is liquid/air surface tension. The Reynolds number calculated for our observed finger velocities, $10 \sim$ $10^{2} \mathrm{~cm} \mathrm{~s}^{-1}$, is in the range of 10 to 100 ; therefore, the transient finger motion is approximately laminar. If the usual no-slip condition on the liquid/dielectric interface is assumed, the viscous force is

$$
F_{v}=-(2 w+g) z \tau_{z}
$$


where $\tau_{z}$, the viscous shear stress, is proportional to the transverse velocity gradient at the substrate surface and the liquid viscosity $\mu$ :

$$
\tau_{z}=\mu \cdot \frac{\partial v_{z}}{\partial x} .
$$

Using a conformal mapping transformation, the velocity profile is [13]

$$
\frac{\partial v_{z}}{\partial x}=\frac{2}{c(w+g / 2)} \cdot \frac{\mathrm{d} z}{\mathrm{~d} t}
$$

where $c=0.507$ is a dimensionless constant obtained from conformal mapping and numerical integration. Then, the viscous drag force becomes

$$
F_{v}=-\frac{4 \mu}{c} z \frac{\mathrm{d} z}{\mathrm{~d} t}
$$

The contact line friction force $F_{\mathrm{cf}}$, thought to be associated with energy dissipation caused by molecular kinetics, is proportional to the product of the transverse projected width of the contact line and its velocity $\mathrm{d} z / \mathrm{d} t[14,15]$ :

$$
F_{\mathrm{cf}}=-(2 w+g) \xi \frac{\mathrm{d} z}{\mathrm{~d} t}
$$

where $\xi$ is the contact line friction coefficient. $\xi$, not known in advance, must be determined by fitting the hydrodynamic model to the experimental data [12]. Note that the exact outline of the three-phase contact line does not influence the net force, as expressed in equation (7), because dissipation associated with molecular dynamic processes must be proportional to the rate at which area is covered by the finger.

From these considerations, the transient motion of the liquid finger can be described by the following nonlinear equation of motion:

$$
\begin{aligned}
& \frac{\pi}{2}\left(w+\frac{g}{2}\right)^{2} \rho \frac{\mathrm{d}}{\mathrm{d} t}\left(z \frac{\mathrm{d} z}{\mathrm{~d} t}\right)+\frac{4 \mu}{c} z \frac{\mathrm{d} z}{\mathrm{~d} t}+(2 w+g) \xi \frac{\mathrm{d} z}{\mathrm{~d} t} \\
& \quad=\frac{1}{2} \frac{\mathrm{d} C}{\mathrm{~d} z} V^{2} u(t)-\pi\left(w+\frac{g}{2}\right) \gamma
\end{aligned}
$$

where $\mu, \xi$, and $\gamma$ have already been defined, $\rho$ is liquid density and $u(t)$ is the unit step function. The left-hand side of equation (8) includes all terms that depend on finger velocity, namely momentum, viscous drag and contact line friction, respectively. On the right-hand side of equation (8) are the DEP electrical force, applied at $t=0$, and the constant capillary force. Note that the DEP force must overcome the constant capillarity force, which imposes a minimum condition on the applied rms voltage, namely $V>V_{\mathrm{th}}=$ $\sqrt{2 \pi(w+g / 2) \gamma /(\mathrm{d} C / \mathrm{d} z)}$.

\section{Fabrication of microfluidic structures}

The DEP structures are fabricated on commercial $\langle 100\rangle \mathrm{Si}$ wafers (thickness $\sim 550 \mu \mathrm{m}$ ) using standard photolithography. The wafers, with an $8 \mu \mathrm{m}$ tetra-ethyl-ortho-silicate (TEOS) based PECVD $\mathrm{SiO}_{2}$ passivation layer for isolation, are photolithographically patterned with evaporated Al electrodes of $0.25 \mu \mathrm{m}$ thick. These structures are then spin-coated with (i) $\sim 0.55 \mu \mathrm{m}$ of spin-on-glass (SOG) (Futurrex IC1-200) of dielectric constant $\kappa_{s}=4$ and (ii) $\sim 0.1 \mu \mathrm{m}$ of amorphous fluoropolymer (DuPont Teflon-AF) with $\kappa_{t}=1.9$. We find that combining the fluoropolymer and SOG layers forms a

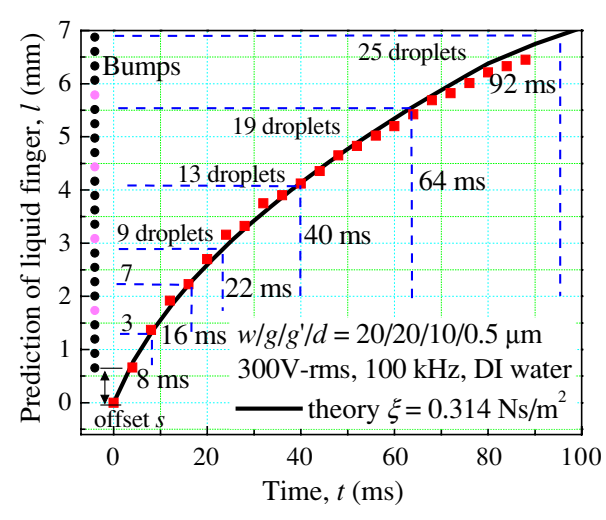

Figure 3. Experimental finger length data, $z(t)$ and superimposed plot of predicted transient motion from equation (1), as fitted to the experimental data using $\xi=0.314 \mathrm{Ns} \mathrm{m}^{-2}$ for contact line friction coefficient. A least squares curve fit using this value yields excellent correlation, $r_{c}=0.99$.

planarized coating system that offers good breakdown (SOG:

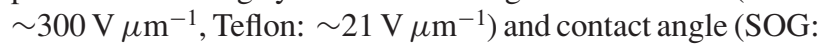
80-85 ; Teflon: $115-120^{\circ}$ ). Another important advantage of $\mathrm{Si}$ is its high thermal conductivity, almost a factor of 10 higher than glass. In DEP actuation using structures fabricated on glass substrates, an oil bath is often needed to ameliorate Joule heating of the liquid. In contrast, no significant heating is observed with Si substrates and the oil layer is unnecessary.

\section{Experiments}

Finger motion was recorded at $500 \mathrm{fps}$ with a high-speed camera (Photron FASTCAM-PCI); selected frames from these videos were processed using a MATLAB ${ }^{\mathrm{TM}}$ program to track the leading edge of the moving finger. A range of electrode dimensions were used: $w / g / g^{\prime}=13 / 9 / 5 \mu \mathrm{m}, 20 / 20 / 10 \mu \mathrm{m}$ and $30 / 30 / 10 \mu \mathrm{m}$ at ac voltages from 250 to $450 \mathrm{v}$-rms and frequency $100 \mathrm{kHz}$. The liquid was DI water: dielectric constant $\kappa_{l}=80$, conductivity $\sigma_{l} \approx 1.5 \times 10^{-4} \mathrm{~S} \mathrm{~m}^{-1}$, surface tension $\gamma=0.073 \mathrm{~N} \mathrm{~m}^{-1}$.

Electronic timer-controlled solid-state relays were used for separate control of the voltages applied to E2 and E3. Refer to figure $1(c)$. The E2 voltage on-time, $t_{\text {trap }}$, determines the length of the finger before it is pinched off at the gap. When this liquid communication is broken, the supply of liquid is cut off within $\sim 1 \mathrm{~ms}$, and finger motion stops. The trapped inventory of liquid along electrode E3 is maintained in electrohydrostatic equilibrium until E3 voltage removal at time $t_{\text {trap }}+\Delta t$, when capillary instability breaks up the trapped liquid into uniformly sized and spaced droplets. The additional time, $\Delta t$, while not critical, must be sufficient to assure that the finger is severed cleanly at the gap $g^{\prime}$ and that the distribution of liquid is equalized along entire trapped length of the finger.

Figure 3 plots experimental data for the number of DI water droplets dispensed, which is directly proportional to finger length, versus the preset on-time $t_{\text {trap }}$ for a structure with dimensions $w / g / g^{\prime}=20 / 20 / 10 \mu \mathrm{m}$. The applied voltage was $V=300( \pm 30)$ V-rms at $100 \mathrm{kHz}$, and the calculated threshold voltage is $V_{\text {th }}=221 \mathrm{~V}$-rms. The circles along the left vertical axis mark the actual positions of the droplet-forming bumps. Because the parent drop was manually placed, there was a 


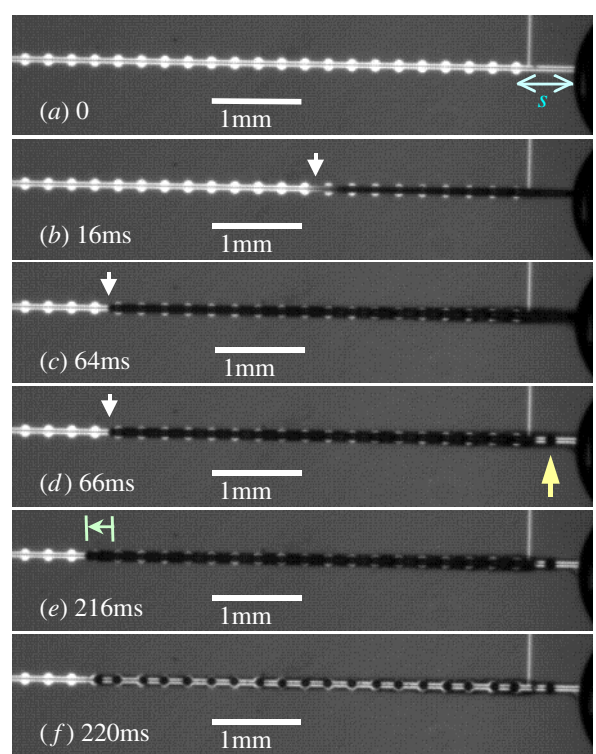

Figure 4. DEP finger actuation and time-based droplet metering on the three-electrode structure. (a) Manually dispensed parent drop (at far right) to right of T-junction gap at distance $s$ from the first bump; (b) - (c) liquid finger at $z \approx 5.5 \mathrm{~mm}$ covers 18 bumps within $64 \mathrm{~ms}$; $(d)$ at $t=66 \mathrm{~ms}$, voltage removed from electrode E2 breaks finger from parent drop and traps liquid along E3; $(e)$ by $t=216 \mathrm{~ms}$, finger has spread out to cover one additional bump, presumably due to the equalization of the finger profile; $(f)$ removal of voltage from E3 at $t=220 \mathrm{~ms}$ forms 19 droplets of volume $380 \mathrm{pl}$.

$0.65 \mathrm{~mm}$ offset for the front edge of the parent drop from the first bump. The results indicate that the gap $g^{\prime}$ near the $\mathrm{T}$-junction has a negligible retarding effect on the finger dynamics.

The solid curve in the figure is obtained from a solution of equation (1) using $\xi=0.314 \mathrm{Ns} \mathrm{m}^{-2}$ for the contact line coefficient, with a correlation coefficient of $r_{c}=0.99$, which is reasonably consistent with our previous work [12]. The same curve fitting performed for other data sets gives values of $\xi$ in the range from 0.1 to $0.5 \mathrm{Ns} \mathrm{m}^{-2}$. The open-loop control strategy for repeatable dispensing of a predetermined number of droplets hinges on this strong correlation of voltage on-time to be required to dispense the desired number of droplets.

DEP actuation and droplet dispensing can usually be repeated on the same structure as many as ten times, after which the Teflon coating apparently deteriorates. We believe this limitation can be circumvented by using a dip-coating process to apply the Teflon. Figure 4 is a selection of video images revealing finger actuation and droplet formation achieved with the open-loop dynamic control scheme. The initial velocity of the leading edge, marked by a white arrow (figures $4(b)-(d)$ ), was $\sim 200 \mathrm{~mm} \mathrm{~s}^{-1}$. The timer controlling the voltage on E2 was preset to $t_{\text {trap }}=64 \mathrm{~ms}$, corresponding to a predicted finger length of $5.6 \mathrm{~mm}$ or 19 droplets, as shown in figure 3. By lapsed time $t=64 \mathrm{~ms}$, the finger, now slowed to $\sim 50 \mathrm{~mm} \mathrm{~s}^{-1}$, had covered 18 bumps (figure 4(c)), one short of the predicted value of 19 . Figure $4(d)$, taken at $t=66 \mathrm{~ms}$, shows that the liquid has been trapped along E3. Note an extraneous droplet formed along E2. The timer controlling the voltage on E3 was preset so that $\Delta t=156 \mathrm{~ms}$, far longer than necessary. In figure 4(e), taken at $t=216 \mathrm{~ms}$ and with the E3 voltage still on, the trapped

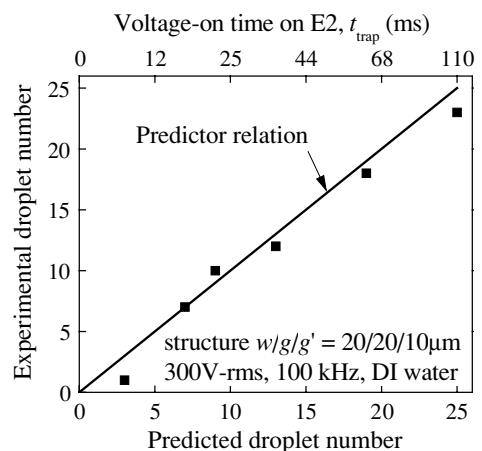

Figure 5. Experimental droplet metering (black squares) and model-based prediction (solid line). The number of droplets is consistent to prediction within an error of \pm 2 droplets. Note the timescale on top showing the range of $t_{\text {trap }}$ values is not linear because the finger steadily decelerates as its length, a consequence of the influence of viscous drag on the motion as reflected in the equation of motion.

liquid had extended a short distance (leftward arrow) and covered one additional bump, presumably due to adjustment of the hydrostatic equilibrium. As shown in figure 4( $f$ ), when voltage was removed from E3 at $t=t_{\text {trap }}+\Delta t=220 \mathrm{~ms}$, the trapped finger broke up into 19 droplets, each of volume $\sim 380 \mathrm{pl}$.

Systematic tests were performed with these structures to investigate the performance of the time-based droplet dispensing scheme. Figure 5 shows data for a wide range of settings for the E2 voltage on-time $t_{\text {trap }}$, from $8 \mathrm{~ms}$ to $110 \mathrm{~ms}$. This range yielded from 1 to 23 uniform droplets in a fashion is reasonably consistent with the predictor relation based on the solution of equation (1).

\section{Conclusion}

We have achieved reproducible, DEP-actuated, controllable formation of multiple aqueous droplets on a Si substrate by employing a novel three-electrode structure and controlling the voltage on-time, $t_{\text {trap }}$ with a preset electronic timer. The open-loop control scheme is based on a predictor relation derived from a simple, nonlinear dynamic model for the finger dynamics. Critical to the success of the model is reliable quantitative knowledge of the dynamic contact line friction coefficient, which must be determined by curve fitting. The three-electrode device traps a controlled volume of liquid along the active section of the structure, avoiding capillary-driven back flow. For the structures tested, reproducible formation of from 1 to 23 uniform droplets ranging in volume from $\sim 75 \mathrm{pl}$ to $\sim 1.3 \mathrm{nl}$ has been demonstrated. With parallel arrays of these co-planar electrode structures, it should be possible to form up to $10^{3}$ uniform, sessile droplets at a time in $\sim 200 \mathrm{~ms}$. The capability to form such large arrays of analyte or sample droplets on an open substrate presents a new opportunity for the application of liquid DEP microfluidics in the laboratoryon-a-chip.

The most important result of this research is that the transient, DEP driven microfluidic flow, while very rapid, is regular and predictable. The small discrepancies encountered in the number of droplets produced versus the number 


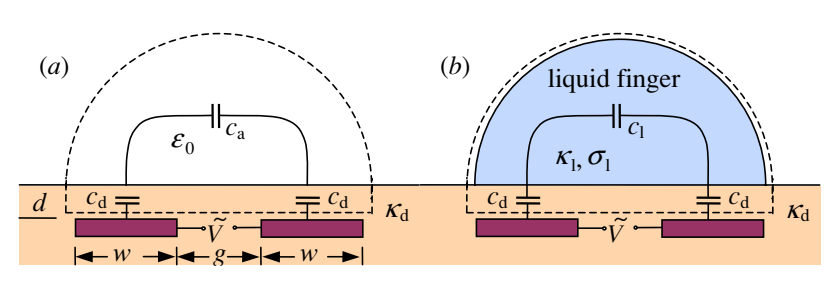

Figure 6. Cross-sectional views of the coplanar electrode structure with $w=$ width and spacing $=g$ without finger $(a)$ and with covering liquid finger $(b)$. The thinness of the dielectric layer $d \ll$ $w, g$ helps establish the condition for a uniform, semicircular finger profile. The two circuit models are used to obtain the required expression for the electromechanical system capacitance $C(z)$, where $z$ is the time-dependent finger length. This model is adequate at high frequencies where the conductivity of the liquid may be ignored.

commanded by the preset on-time $t_{\text {trap }}$ can probably be eliminated by a small number of optical or capacitive sensors per structure that detect the finger as it passes fixed points along the structure. The outputs from these sensors would be used to correct for minor performance variations by implementing a very simple, closed-loop control scheme.

\section{Acknowledgments}

Tina Prevost of RIT fabricated the chips used in the experiments. This work was supported by grants from the Infotonics Technology Center (Canandaigua, NY) and the National Science Foundation (USA). Additional support from the Electrical and Computer Engineering Department of the University of Rochester is acknowledged.

\section{Appendix}

The net force of electrical origin may be determined using lumped parameter electromechanics. To employ this method, an expression for the total capacitance of the system within the volume $\Sigma$ is required. This capacitance must depend on a mechanical variable, which in the present case is the finger length, $z$. We assume that the semicircular profile of the liquid finger is uniform along its length and constant in time. Figures $6(a)$ and $(b)$ show cross sections of the structure without and with the liquid finger with conceptual capacitive circuit elements. We also assume the frequency is high enough $(>50 \mathrm{kHz})$ so that the conductivity of the liquid does not influence the voltage distribution. If the thickness of the dielectric layer $d$ is much smaller than the electrode width $w$ and spacing $g$, the capacitances per unit length attributed to the dielectric layer, the half-space of air and the semicircular cross section of liquid are approximately $c_{d}=\kappa_{d} \varepsilon_{0} w / d, c_{a}=\varepsilon_{0} A$, and $c_{l}=\kappa_{l} c_{a}$, respectively. Here, $A=I\left(1-k^{2}\right) / 2 I\left(k^{2}\right)$ is a geometric factor and $I\left(k^{2}\right)$ is the complete elliptical integral of the first kind with modulus $k=g /(2 w+g)$ [16]. The total capacitance $C$ in the control volume is then

$$
C(z)=\frac{z}{1 / \kappa_{l} c_{a}+2 / c_{d}}+\frac{l-z}{1 / c_{a}+2 / c_{d}} .
$$

The ac voltage applied to the electrodes is $\tilde{V}(t)=$ $\operatorname{Re}\left[\sqrt{2} V \mathrm{e}^{\mathrm{j} \omega t}\right]$, where $\omega$ is the radian frequency and $V$ is the rms value. Standard energy methods from lumped parameter electromechanics yield a free energy function, called the coenergy [17], for the system enclosed by the control volume: $W_{e}^{\prime}=C(z) V^{2} / 2$, from which the net driving force of electric origin may be determined.

$$
F^{e}=\left.\frac{\partial W_{e}^{\prime}}{\partial z}\right|_{V=\text { constant }}=\frac{1}{2} \frac{\mathrm{d} C}{\mathrm{~d} z} V^{2} .
$$

A very clear advantage of this lumped parameter force formulation is that it obviates any need to consider the local details of the electric field or contact angle at the leading edge of the moving finger.

\section{References}

[1] Prins M W J, Welters W J J and Weekamp J W 2001 Fluid control in multichannel structures by electrocapillary pressure Science 291 277-80

[2] Duffy D C, Schueller O J, Brittain O J A and Whitesides G M 1999 Rapid prototyping of microfluidic switches in poly(dimethyl siloxane) and their actuation by electro-osmotic flow J. Micromech. Microeng. 9 211-7

[3] McBride S E, Moroney R M and Chiang W 1998 Micro Total Analysis Systems ed D J Harrison and A Van Den Berg (Dortrecht, NL: Kluwer) pp 45-8

[4] Sondag-Huethorst J A M and Fokkink L G J 1994 Electrical double-layers on thiol-modified polycrystalline gold electrodes J. Electroanal. Chem. 367 49-57

[5] Pollack M G, Fair R B and Shenderov A D 2000 Electrowetting-based actuation of liquid droplets for microfluidic applications Appl. Phys. Lett. 77 1725-6

[6] Jones T B, Gunji M, Washizu M and Feldman M J 2001 Dielectrophoretic liquid actuation and nanodroplet formation J. Appl. Phys. 89 1441-8

[7] Jones T B 2005 An electromechanical interpretation of electrowetting J. Micromech. Microeng. 15 1184-7

[8] Ahmed R and Jones T B 2006 Dispensing picoliter droplets on substrates using dielectrophoresis J. Electrost. 64 543-9

[9] Rayleigh J W S 1945 The Theory of Sound vol 2 (New York: Dover) p 359

[10] Schiaffino S and Sonin A A 1997 Formation and stability of liquid and molten beads on a solid surface J. Fluid Mech. 343 95-110

[11] Blake T D and Haynes J M 1969 Kinetics of liquid/liquid displacement J. Colloid Interface Sci. 30421

[12] Wang K-L and Jones T B 2005 Electrowetting dynamics of microfluidic actuation Langmuir 21 4211-7

[13] King M R, Lomakin O A, Ahmed R and Jones T B 2005 Size-selective deposition of particles combining liquid and particulate dielectrophoresis J. Appl. Phys. 97054902

[14] Decamps C and DeConinck J 2000 Dynamics of spontaneous spreading under electrowetting conditions Langmuir 16 10150-3

[15] Ren H, Fair R B, Pollack M G and Shaughnessy E J 2002 Dynamics of electro-wetting droplet transport Sensors Actuators B 87 201-6

[16] Jones T B 2001 Dynamics of Dielectrophoretic Liquid Microactuation 4th Int. Conf. on Applied Electrostatics (Dalian, China)

[17] Woodson H H and Melcher J R 1968 Electromechanical Dynamics (Part I) (New York: Wiley) chapter 3 